\title{
Correction to Intuitive Eating Scale-2: psychometric properties and clinical norms among individuals seeking treatment for an eating disorder in private practice
}

\author{
Katie M. Babbott ${ }^{1}$ D $\cdot$ Deborah Mitchison ${ }^{2,3} \cdot$ Chris Basten $^{3} \cdot$ Chris Thornton $^{4} \cdot$ Phillipa Hay $^{6} \cdot$ Sue Byrne $^{8}$. \\ Mandy Goldstein $^{5,6} \cdot$ Gabriella Heruc $^{6,7} \cdot$ Bert van der Werf $^{9} \cdot$ Nathan S. Consedine ${ }^{10} \cdot$ Marion Roberts $^{1,11}$
}

Published online: 12 January 2022

(c) Springer Nature Switzerland AG 2022

\section{Correction to: \\ Eating and Weight Disorders-Studies on Anorexia, Bulimia and Obesity https://doi.org/10.1007/s40519-021-01326-x}

The Table 1 was in correctly published in the original publication. The complete correct Table 1 is given below.

The original article can be found online at https://doi.org/10.1007/ s40519-021-01326-x.

Katie M. Babbott

katie.babbott@auckland.ac.nz

Deborah Mitchison

Deborah.Mitchison@westernsydney.edu.au

Chris Basten

ChrisB@bastenpsychology.com.au

Chris Thornton

chris.thornton@theredleafpractice.com

Phillipa Hay

p.hay@westernsydney.edu.au

Sue Byrne

sue.byrne@uwa.edu.au

Mandy Goldstein

mandy.goldstein@mgpsych.com

Gabriella Heruc

gheruc@appetiteforchange.com.au

Bert van der Werf

bert.vanderwerf@auckland.ac.nz

Nathan S. Consedine

n.consedine@auckland.ac.nz

Marion Roberts

m.roberts@auckland.ac.nz
1 General Practice and Primary Healthcare, School of Population Health, Faculty of Medical and Health Sciences, University of Auckland, Private Bag 92019, Auckland, New Zealand

2 Translational Health Research Institute, School of Medicine, Western Sydney University, Sydney, Australia

3 Department of Psychology, Macquarie University, Sydney, Australia

4 The Redleaf Practice, Sydney, Australia

5 Mandy Goldstein Psychology, Sydney, Australia

6 School of Medicine, Western Sydney University, Campbelltown, Australia

7 Appetite for Change, Sydney, Australia

8 University of Western Australia, Perth, Australia

9 Department of Biostatistics, University of Auckland, Auckland, New Zealand

10 Department of Psychological Medicine, Faculty of Medical and Health Sciences, University of Auckland, Auckland, New Zealand

11 Nurture Psychology, Auckland, New Zealand 
Table 1 Demographic characteristics of the sample

\begin{tabular}{|c|c|}
\hline$N$ & 569 \\
\hline \multicolumn{2}{|l|}{ Gender, $n(\%)$} \\
\hline Female & $542(95.3 \%)$ \\
\hline Male & $24(4.2 \%)$ \\
\hline Non-binary & $3(0.5 \%)$ \\
\hline \multicolumn{2}{|l|}{ Age, mean (SD) } \\
\hline Years & $23.5(9.3)$ \\
\hline \multicolumn{2}{|l|}{ Country of origin, $n(\%)$} \\
\hline Australia & $422(74.2 \%)$ \\
\hline Europe & $44(7.7 \%)$ \\
\hline Asia & $18(3.2 \%)$ \\
\hline Africa & $8(1.4 \%)$ \\
\hline North America & $8(1.4 \%)$ \\
\hline Other Pacific & $6(1.1 \%)$ \\
\hline New Zealand & $52(9.1 \%)$ \\
\hline Other & $6(1.1 \%)$ \\
\hline N.S & $3(0.6 \%)$ \\
\hline \multicolumn{2}{|l|}{ Cultural identity, $n(\%)$} \\
\hline Australian Aboriginal & $4(0.7 \%)$ \\
\hline New Zealand Māori & $3(0.5 \%)$ \\
\hline Pacific Islander & $3(0.5 \%)$ \\
\hline N.S & $559(98.3 \%)$ \\
\hline \multicolumn{2}{|l|}{ Diagnosis, $n(\%)$} \\
\hline Anorexia nervosa (binge/purge) & $27(4.7 \%)$ \\
\hline Anorexia nervosa (restrictive) & $94(16.5 \%)$ \\
\hline Bulimia nervosa & $67(11.8 \%)$ \\
\hline Binge eating disorder & $41(7.2 \%)$ \\
\hline Avoidant/restrictive food intake disorder & $5(0.9 \%)$ \\
\hline OSFED: anorexia nervosa ${ }^{a}$ & $73(12.8 \%)$ \\
\hline OSFED: bulimia nervosa ${ }^{\mathrm{b}}$ & $12(2.1 \%)$ \\
\hline OSFED: binge eating disorder ${ }^{b}$ & $10(1.8 \%)$ \\
\hline OSFED: purging disorder & $7(1.2 \%)$ \\
\hline Unspecified feeding or eating disorder & $21(3.7 \%)$ \\
\hline Body dysmorphic disorder & $2(0.4 \%)$ \\
\hline In remission & $5(0.9 \%)$ \\
\hline N.S & $205(36.0 \%)$ \\
\hline
\end{tabular}

N.S. not specified, OSFED other specified feeding or eating disorder

${ }^{\mathrm{a}}$ Typical, ${ }^{\mathrm{b}}$ of low frequency and/or limited duration
Publisher's Note Springer Nature remains neutral with regard to jurisdictional claims in published maps and institutional affiliations. 